\title{
Design of Medical Sealing Machine Based on ARM Processor Jian $\mathrm{Li}^{1}$, Xiaojuan $\mathrm{Li}^{1}$ and Jiani $\mathrm{Liu}^{1}$ \\ ${ }^{1}$ Jilin Agricultural University, Changchun 130118, Jilin Province, China
}

\begin{abstract}
For the hospital disinfection center, operating room and dentistry and other medical equipment after sterilization sealed package to provide a perfect solution.ARM as the core processor, to control closed-loop temperature sensing heating system, through the capacitive touch LCD screen using graphical interface for human-computer interaction. Driven by the DC motor, through the gear and belt drive system, driven paper plastics bags to move, to achieve medical paper plastic disinfection bags of continuous automated sealing printing. Medical sealing machine for two, three types of medical equipment packaging and heat sealing, sealed transparent composite mold (PETP / PE), special Wei strong paper (TYVEK). Parts and are more usedthe aluminum, with a reasonable structure design, to achieve the maximum reduction of the weight of the machine.
\end{abstract}

Keywords: Medical equipment; Sealing machine; Medical packaging paper bag; Arm; Mechanical linkage

\section{基于 ARM 处理器医用封口机的设计}

\author{
李健 ${ }^{1}$, 李小娟 ${ }^{1}$, 刘佳妮 ${ }^{1}$ \\ ( ${ }^{1}$ 吉林农业大学, 吉林长春 130118)
}

摘要: 针对医院消毒中心、手术室和口腔科等医疗器械灭菌后的密封包装提供完美的解决方案。以 ARM 为核心处理器, 控制闭环温度传感加热系统, 通过电容触摸液晶屏采用图形界面进行人机交互。采用直流电机驱动, 通过齿轮和皮带传动系 统, 带动纸塑袋移动, 实现医用纸塑消毒袋的连续自动化封口打印。医用封口机适用于二类、三类医疗器械包装及热封, 可 密封透明复合模 ( PETP/PE)、特卫强纸 (TYVEK)。零部件多采用铝材, 配合合理的结构设计, 最大限度的降低了整机重 量。

关键字: 医疗器械; 封口机; 医用包装纸袋; ARM处理器; 机械联动

\section{1 引言}

纸塑包装袋近年来已经由新型包装材料成为无菌物品的常用包装材料之一，它具有良好的生物屏障作 用, 使灭菌物品的保存期较传统的包装的灭菌有效期长, 同时具有其美观可视性, 已广泛运用于医院消毒 灭菌品的包装。但由于封装操作过程中的温度、速度等控制不当, 使得封口不紧或裂开, 包装的闭合性达 不到应有的“阻菌”效果 [1]。目前使用的医用封口主要有德国合福 HM850 DC-V 型医用封口机, 美国 AccuSeal 医用封口机，日本富士 OPL-350-MDNP 医用封口机。国产品牌主要是山东逸风医疗科技有限公司、青岛秀 海医疗器械有限公司等生产的医用封口机。

\section{2 系统结构}

本医用封口机是采用多种新材料、新元件及高新技术研发出来的一种新型产品，具有技术先进、造型 美观、结构紧凑、品质优良、使用安全无噪音、清洁保养方便等一系列特点, 将逐步成为各地区畅销产品。 


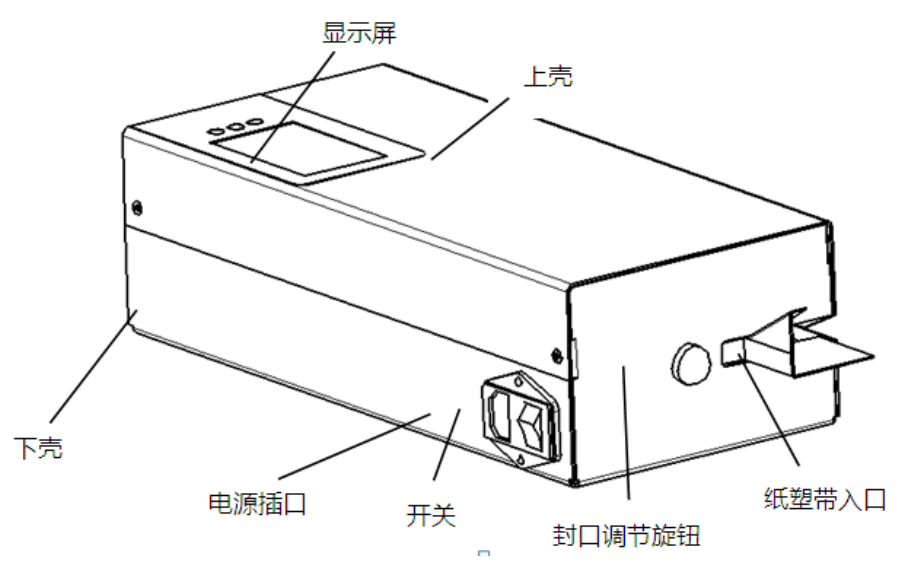

图 1 封口机外观标识图

医用封口机包括动力源驱动、传动机构和控制系统组成。适用于二类、三类 PVC、TPU 等材料医疗器 械包装及热封的各种医用袋产品。如: 医用输液袋、输血袋、导尿袋、冲洗袋、引流袋等一次性医疗用品 袋。其技术参数如表 1 所示。

表 1 技术参数

\begin{tabular}{|l|l|}
\hline 封印速度 & $10 \pm 0.1 \mathrm{~m} / \mathrm{min}$ \\
\hline 封印温度 & $90-200^{\circ} \mathrm{C}$ \\
\hline 控温精度 & $\leqslant \pm 1 \%$ \\
\hline 封纹宽度 & $12 \mathrm{~mm}$ \\
\hline 封口留边 & $0 \sim 35 \mathrm{~mm}$ 可调 \\
\hline 外形尺寸 & $365 \times 163 \times 103 \mathrm{~mm}$ \\
\hline 交流电源: & $220 \mathrm{~V} 50 \mathrm{~Hz}$ \\
\hline
\end{tabular}

功能特性

1)、采用 7 寸彩色液晶触摸屏，图像化界面操作简单，显示清楚;

2)、自动化封口速度 $10 \pm 0.1 \mathrm{~m} / \mathrm{min}$ ，封口效率高;

3)、采用浮动式恒定压力压合结构设计，适用立体袋和不同厚度纸袋的封口需要;

4)、先进的陶瓷加热组件, 可干烧、耐高温、寿命长、热效率高; 嵌入式智能温度控制设计, 工作温 度 $60 \sim 220^{\circ} \mathrm{C}$ 任意设置, 温控精度 $\pm 1 \%$, 升温快、加热均匀;

5)、内置一台微型打印机, 中文、英文、数字以及符合《YYO466-2003 医疗器械用于医疗器械标签、 标记和提供信息的符号》等特殊符号打印功能, 可实现卫生部要求的打印灭菌日期 (封口日期)、有效期、 打印封口机编码、包装卷料批号、操作者姓名代码、锅号、锅次、医院科室名称、CE 认证及 EN980 标准的 标识等各种打印要求; 打印起始边距可调, 打印标徽可旋转 $180^{\circ}$, 字体可选, 字距可调, 打印功能可一 键关闭也可按需要关闭某条目; 打印内容与纸袋宽度智能辅助匹配, 即系统会根据选择的打印内容给出纸 袋宽度要求, 操作者确定打印内容或选取合适的纸袋; 纸袋宽度不足时报告提醒。灭菌日期、失效日期根 据设置自动进行调整，不需手动调整大小月天数;

6)、温度保险丝、温度断路器等多重安全保护。不锈钢面板, 外观简洁易于清洁和清毒。本机器驱动 
部分采用 $24 \mathrm{~V}$ 的直流减速电机作为动力源。直流减速电机结构紧凑, 体积小, 造型美观, 承受过载能力强。 同时使用专为此医用封口机制造的运转平稳的低噪音电机, 从动力输出源头控制了机器的振动和噪音, 从 而提升整机的噪音表现。

传动部分动力从电机输出后, 先经过一对雉齿轮传递给机器主体。采用锥齿轮可以更好地利用机器内 部的空间, 使电机和机器主体平行放置, 结构更加紧凑。雉齿轮的材料采用尼龙, 尼龙齿轮的优点是重量 轻、耐疲劳、吸收冲击、自润滑性好, 对降低噪音也有明显作用。通过一对尼龙直齿轮将动力分配到上下 两轴。上轴有两套皮带传动系统, 一套带动压紧轮 (属于功能部分), 另一套和下轴的皮带系统配合工作, 带动由入口处进入的纸塑袋移动。下轴则只有一套皮带传动系统。加热棒是由铝棒和内部的加热管组成的, 由皮带组带动的纸塑带经过加热区域时升温至密封温度。压紧轮分上下两个, 上轮由上轴的皮带带动旋转, 下轮通过可调压紧机构随上轮同步旋转。当纸塑带通过加热区后, 由压紧轮压合封口。通过旋钮可以调节 封口边距，适应不同用户需求。加热棒支架除了固定加热棒，限定位置外，底部弹簧可以让上下加热棒既 可以充分加热纸塑带, 又不会因压力太大造成卡纸。运送皮带导轨采用一边弹簧式的导轨, 使得上下皮带 间保持一定的夹紧力，带动纸塑带前进。

控制系统中 STM32F407ZGT6 处理器采集加热棒的温度参数, 根据触摸屏的输入设置控制电机的转速、 封口温度和打印机等, 通过液晶屏进行输出显示。内置时钟具有自动存储及自动待机功能, 最大限度节约 能源, 保证设备性能。

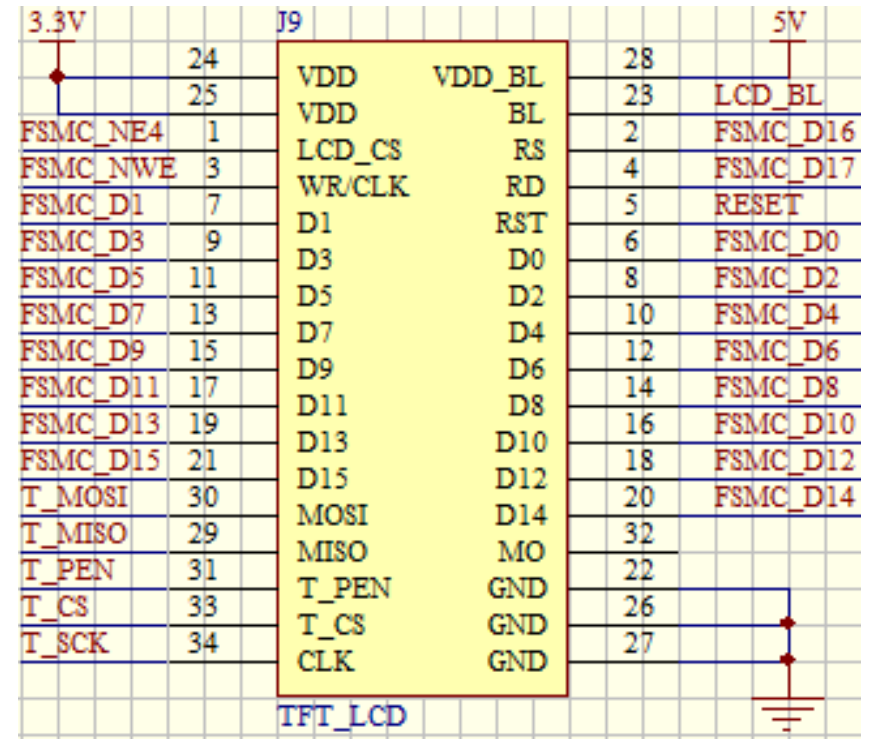

图 2 液晶触摸屏接口图

7 寸 TFTLCD 模块连接在 STM32F407ZGT6 的 FSMC 总线上面, 显著调高 LCD 的刷屏速度。图中 T_MISO、 T_MOSI、T_PEN、T_CS、T_SCK 用来实现对电容触摸屏的控制。LCD_BL 控制 LCD 的背光。复位信号 RESET 和处理器共用一个复位电路。

\section{3 实验}

打开电源开关进行预热，液晶屏上显示预热状态。按触摸屏上 SET 键进入温度设定界面，通过 键调整到需要的封口温度, 再次按 SET 退回到主界面。预热到设定温度（出厂设定的封口温度为 $200^{\circ} \mathrm{C}$ ) 
后语音和图标提示, 电机开始运转, 液晶屏显示为工作状态, 从入口处放入需要封口的纸塑带即可完成自 动封口。通过调节入口旁的旋钮可以调节封口到边缘的距离, 选用德国联合纸业有限公司的 Stericlin 牌 包装纸袋、上海建中医疗器械包装有限公司的恩帕克牌纸塑袋, 在不同温度和封口速度下进行封口试验, 将封好的包装袋经 $132^{\circ} \mathrm{C}$ 预真空压力蒸汽灭菌, 灭菌冷却 15 分钟后肉眼检查封口情况。以包装袋灭菌处理 后封口自动裂开、器械与外界相通、封口处起皱、封口的密封带宽度达不到 $6 \mathrm{~mm}$ 、纸面焦化为不合格。经 实验表明封口的成功率取决于封口温度与走纸的速度。用于高温灭菌和环氧乙烷灭菌的纸塑袋, 由三层材 料组成, 最底层医学级皱纹纸、中间聚丙烯 (熔点 $160^{\circ} \mathrm{C}$ ) 、面层聚醋 (熔点 $260^{\circ} \mathrm{C}$ ), 聚醋覆盖保证连续性 封合的可靠性, 聚丙烯覆盖纸面避免气泡效应, 此种袋子选择 $175^{--}-180^{\circ} \mathrm{C}$ 即可。用于低温等离子灭菌的 “特 为强”袋应选择 $125^{\circ} \mathrm{C}$ 。封口强度符合 YY/T0698. 5-2009 的要求。

\section{4 总结}

该机是在吸取国内外同类产品先进技术的基础上经过精心研发创新设计而成，它以机械联动机构以为 主体, 采用 STM32F407ZGT6 型 ARM 处理器进行闭环控制, 温度、压力等封口参数可以进行调节, 具有运行 稳定可靠、封口牢固、平整美观等特点。该设备的研制有利于医疗器械领域的科学研究、技术创新与开发、 科技成果推广等方面取得更大的成功。它是一种既能简化加工工艺, 又能达到传动精度的新型封口机。适 用于消毒供应中心、手术室、药品厂家及工业领域等。可对物品进行自动包裹、热封、计数。在更换少量 零件的情况下, 可包装不同规格的纸塑袋。

\section{参考文献}

[1]屈玲．温度与速度在对医用纸塑包装袋封口的影响 [J]．《中国民族民间医药》，2012, 21(13)：69-69.

[2]陈凌晖, 㸚讳升, 李莹. 纸塑包装封口失败原因探论 [J]. 中国消毒学杂志, 2008, 25(2) :194-195.

[3] 黄浩、陈慧. 医用坡纹纸与全棉布包装材料的性能观察 [J].中国消毒学杂志. 2010, 27 (6) : 680-681.

[4]周群、俞星. 纸塑包装高压灭菌器械在口腔科的临床应用 [J]. 实用临床医学. 2008, 9 (9):92.

[5]黄小虹. 纸塑包装无菌物品在临床的应用 [J]. 现代护理、2006, 12 (24) : 2322-2323.

[6] 卓娅、刘志红. 环氧乙烷气体灭菌纸塑包装器物效果观察 [J]. 郑州大学学报、2008、43(4):853-854.

[7] 陈春莲、李玉平、赖秀梅. 棉布与医用立体纸袋包装材料灭菌效果及成本分析 [J]. 现代医院、2010、13(11) : 118-119.

[8] 常香远、陈远芳、郝淑芹. 供应室诊疗包不同包装材料灭菌效果及成本分析 [J]。护理管理研究、2008、 2 (12) : 3264-3265.

[9]赵文颖、刘素哲、刘翠霞等. 医用灭菌纸袋包装无菌物品成本分析川. 护理实践与研究、2011、13:250-251.

[10] 吴秋菊．医院纸塑包装易出现的问题及对策 [J].中国消毒杂志、2010、27 (4) :492-493.

[11]王风琴、牛河裹．纸塑器械袋塑封失败的原因分析 [J]. 中国消毒杂志、2005、22(2) :230-230.

[12] 鲍菲、招彩铃、吕洁梅等. 不同灭菌干燥时间及装载方法对纸塑包装物品灭菌后的影响 [J].中国医院感染学杂志、 2010、20 (3) : 360-361.

\section{References}

[1] Qu Ling. Effect of temperature and speed on sealing of medical paper and plastic bags [J]. "Chinese National Folk Medicine", 2012, 21 (13): 69-69.

[2] Chen Linghui, Tan taboo rose, Li Ying. Journal of Chinese Sterilization, 2008, 25 (2): 194-195. [J]. Chinese Journal of Disinfection, 2008, 25 (2): 194-195.

[3] Huang Hao, Chen Hui. Medical wrinkled paper and cotton cloth packaging materials 
performance observation [J]. Chinese Journal of Disinfection. 2010, 27 (6): 680-681.

[4] Zhou Qun, Yu Xing. Packaging autoclave in the clinical application of dentistry [J]. Practical Clinical Medicine. 2008, 9 (9): 92.

[5] Huang Xiaohong. Packaging sterile items in clinical application [J]. Modern Nursing, 2006, 12 (24): 2322-2323.

[6] ZhuoYa, Liu Zhihong. Observation on the effect of ethylene oxide gas sterilization paper plastic packaging [J]. Journal of Zhengzhou University, 2008, (4): 853-854.

[7] Chen Chunlian, Li Yuping, Lai Xiumei.Therling and medical three - dimensional paper bag packaging material sterilization effect and cost analysis [J]. Modern Hospital, 2010, 13 (11): 118-119.

[8] Chang Xiangyuan, Chen Yuanfang, HaoShuqin. Analysis of sterilization effect and cost of different packaging materials in the supply room. [J]. Nursing Management Research, 2008, 2 (12): 3264-3265.

[9] Zhao Wenying, Liu Suzhe, Liu Cuixia and so on. Cost Analysis of sterile articles in medical sterile paper bags. Nursing Practice and Research, 2011, 13: 250-251.

[10] Wu Qiuju. Hospital paper and plastic packaging problems prone and countermeasures [J]. Chinese Journal of Disinfection, 2010, 27 (4): 492-493.

[11] Wang Fengqin, Niuhe Xiang. Analysis of the causes on failure of plasticization of paper and plastic boxes [J]. Chinese Journal of Disinfection, 2005, 22 (2): 230-230.

[12] BaoFei, ZhaoTieling, LuJie-mei, et al.Effect of different sterilization drying time and loading method on sterilization of paper plastics packaging [J]. Chinese Journal of Nosocomiology, 2010, 20 (3): 360-361. 